\title{
Dynamic 'Spot Sign' Resolution following INR Correction in a Patient with Warfarin-Associated Intracerebral Hemorrhage
}

\author{
$\begin{array}{llll}\text { S. Chakraborty } & \text { G. Stotts } & \text { C. Rush } & \text { M.J. Hogan }\end{array}$ \\ D. Dowlatshahic
}

a Department of Radiology (Neuroradiology), and beurology Resident, University of Ottawa, and 'Department of Medicine (Neurology), University of Ottawa and Ottawa Hospital Research Institute, Ottawa, Ont., Canada

\section{Key Words}

Spot sign · CT angiography · Intracerebral hemorrhage

\begin{abstract}
Hematoma expansion in intracerebral hemorrhage is associated with poor clinical outcome. The 'spot sign' is a radiological marker that is associated with hematoma expansion, and thought to represent active extravasation of contrast. This case demonstrates the use of dynamic CT angiography in identifying the time-dependent appearance of a spot sign in a patient with warfarin-associated intracerebral hemorrhage. Repeat imaging is also presented which verified cessation of the spot sign after INR correction.
\end{abstract}

\section{Introduction}

Intracerebral hemorrhage (ICH) growth occurs early after onset and has been shown to be an independent marker for poor clinical outcome [1-3]. The 'spot sign' is thought to represent contrast pooling within or around a hematoma and can predict future hematoma growth [4]. However, contrary to standard 'static' imaging studies that are acquired at a particular point in time, dynamic CT angiography (dCTA) demonstrates temporal wash-in and wash-out of intravenous contrast material and could capture evolution of spot sign in real time [5]. The success of future ICH therapies, including hemostatic agents, may depend on accurate determination of hematomas at risk of expansion [6, 7]. Here, we present a real-time video (online suppl. video 1, www.karger.com/doi/10.1159/000330304) and a time series of dCTA images

Assist. Prof. Dar Dowlatshahi $M D, P h D, F R C P C$
Department of Medicine (Neurology), University of Ottawa

Ottawa Hospital Research Institute

1053 Carling ave, Ottawa, ON K1Y 4E9 (Canada)

Tel. +1 613761 4709, E-Mail ddowlat@toh.on.ca 
demonstrating spot sign formation from an anticoagulated patient with ICH that resolved after INR correction.

\section{Case Report}

A 63-year-old right-handed female with sudden onset of severe headache and left-sided weakness was brought to the emergency department as a 'stroke code'. She was on long-term anticoagulant therapy (warfarin) due to a history of recurrent, unprovoked thromboembolism for 9 years. On examination, her blood pressure was $136 / 60 \mathrm{~mm} \mathrm{Hg}$ and NIHSS was 8. There was no aphasia or dysarthria and visual fields were intact. There was mild left nasolabial flattening but other cranial nerves were intact. Motor examination showed left hemiplegia with intact sensation. She was anticoagulated with an INR of 3.2.

\section{Scanning Protocol}

Non-contrast CT head images followed by dCTA acquisition were performed using a 320-row volume CT scanner (Aquilion $\mathrm{ONE}^{\mathrm{TM}}$; Toshiba). This system utilizes 320 ultra-high resolution detector rows $(0.5 \mathrm{~mm}$ in width) to image the entire brain in a single gantry rotation. We acquired whole-brain images beginning every $2 \mathrm{~s}$ over a 1-min sequence, and generated real-time flow videos (online suppl. videos 1 and 2) on a Vitrea ${ }^{\mathrm{TM}}$ imaging station for analysis. Hematoma volumes were measured using computerized planimetry. The radiation dose for this study was low with a DLP (dose length product) reading 1,925 mGy-cm compared to a helical CT head scan having a value of 1,349 mGy-cm.

\section{Results}

The admission CT scan of the head showed an acute large right medial frontal parenchymal hemorrhage measuring approximately $8 \times 3 \mathrm{~cm}$ in dimension (igg. 1a). The hemorrhage was heterogeneous with minimal subarachnoid extension and mild perilesional edema. dCTA images demonstrated an increasing area of contrast collection seen along the anterior lateral periphery of the hemorrhage consistent with a spot sign (fig. 2). This does not appear to be directly connected to a vessel. The spot first appeared at $15 \mathrm{~s}$ following start of contrast injection, and steadily increased in size during the remainder of the acquisition. The maximum density was noted around $25 \mathrm{~s}$; after that, the average density decreased with the spot appearing more heterogeneous and the margins became ill-defined, consistent with dispersion of contrast in the hematoma. Baseline hematoma volume was $56 \mathrm{ml}$, and peak spot sign density was 480 Hounsfield units.

\section{Treatment and Follow-Up}

The patient was treated with 2,000 $\mathrm{U}$ (factor IX equivalent) of prothrombin complex concentrate (PCC), which corrected the INR to 1.5. Repeat imaging at $3 \mathrm{~h}$ revealed hematoma expansion of $11.2 \mathrm{ml}$; however, the spot sign was gone on the repeat dCTA study (fig. 1e). Absence of contrast extravasation was also confirmed by conventional angiogram. The final hematoma volume at 3 days was $52 \mathrm{ml}$, indicating hematoma stabilization and resolution (fig. 1f).

The patient had no significant change on neurological in-hospital examination, and a MRI on day 10 confirmed a large right frontal hematoma which appeared stable in size, 
with hemorrhage and perilesional edema extending to the right precentral gyrus explaining her weakness. There were no other underlying abnormalities seen.

Her recovery was complicated by a significant proximal left leg DVT, and therefore, an IVC filter was placed to prevent pulmonary embolism, and she was started on subcutaneous heparin. The patient started to make some gains in her left-sided power by the third week, and she was transferred for rehabilitation therapy with 3/5 power in her left arm and $1 / 5$ power in her left leg.

\section{Discussion}

We described the dynamic evolution of a spot sign by dCTA in a patient with warfarinassociated hemorrhage and its disappearance following INR correction. There was early hematoma expansion following the first CTA, as expected given the elevated INR and presence of a spot sign. However, after PCC administration and INR correction, the spot sign was no longer present, and hematoma volume stabilized.

The spot sign likely represents contrast extravasation into an ICH and has been validated as a predictor for hematoma expansion as well as clinical outcome $[4,6,8,9]$. The CTA spot sign is present in approximately one-third of patients with acute ICH. However, its sensitivity is low, most likely as the detection relies on a single snapshot CT angiographic acquisition $[10,11]$. Extravasation that either begins late, or resolves quickly after contrast injection, may be missed with static CTA. Using the new Aquilion ONE (Toshiba) volume CT scanner, the dynamic evolution of 'spot sign' in an acute hemorrhage was described in real time, in what would have otherwise been called a 'spotnegative' patient [5]. This dynamic property has important clinical implications as hemostatic therapy is likely of minimal benefit in cases where hematoma expansion is not expected [12-14].

The pathophysiology behind the spot sign is not known but it has been proposed that it may relate to underlying Charcot-Bouchard aneurysms or amyloid-related microaneurysms versus active extravasation [4]. The dynamic images clearly show contrast extravasation as the cause of 'spot' in our patient (fig. 2). dCTA may be useful in analyzing the cause(s) of spot sign formation by providing estimations of extravasation rate.

The follow-up dCTA $3 \mathrm{~h}$ after treatment with PCC did not reveal a spot sign. It is possible that this represents the effect of PCC correction and hematoma stabilization. It is also possible that the hematoma may have reached maximal expansion even before PCC was instituted. In either case, this result reveals an important tool for use in monitoring patients by repeat dCTA to determine if there is ongoing extravasation.

\section{Conclusion}

This is the first case report we are aware of involving dCTA documentation of a spot sign that would be difficult to verify by standard imaging techniques. This case also demonstrated hematoma stabilization occurring after INR correction in a warfarin- 
associated hemorrhage. We propose that future studies incorporating the spot sign also examine its dynamic nature.

\section{Acknowledgements}

D.D. is supported by a research grant from the J.P. Bickell Foundation, and a research award from the Department of Medicine, University of Ottawa. S.C., G.S., C.R., and M.J.H. have nothing to declare.
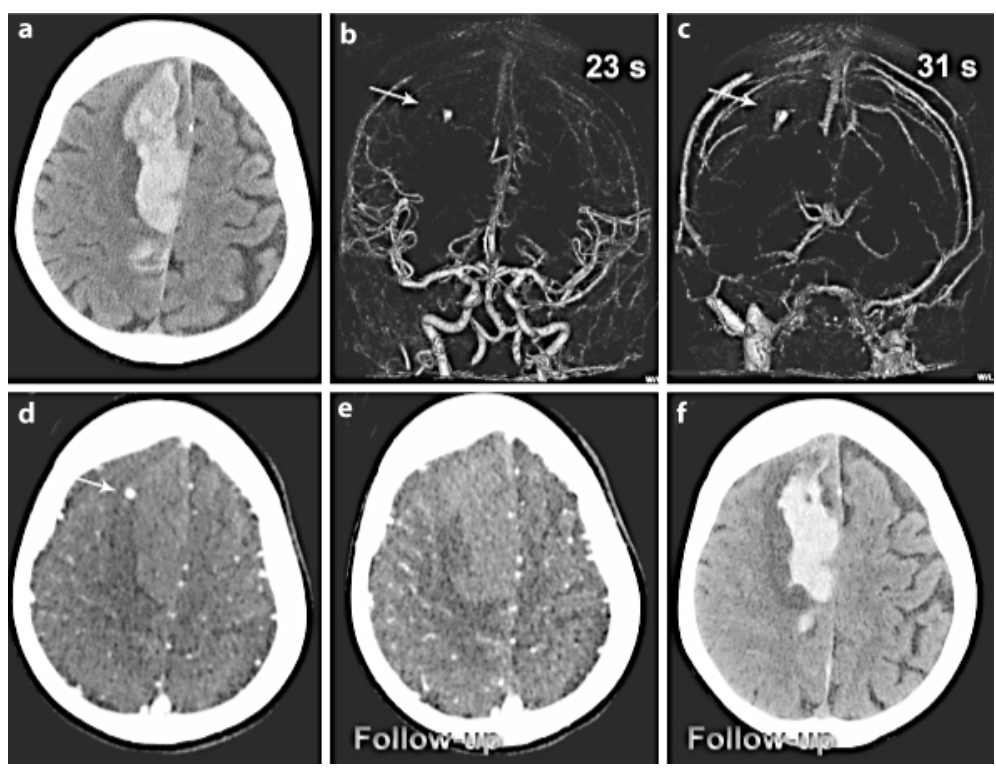

Fig. 1. a Admission non-contrast CT shows a right medial frontal hematoma (volume $56 \mathrm{ml}$ ) and a small amount of subarachnoid blood in the adjacent posterior frontal sulci. Dynamic CT angiography (dCTA) images at $23 \mathrm{~s} \mathrm{(b)} \mathrm{and} 31 \mathrm{~s}$ (c) show an enlarging spot sign. d Axial images from the admission dCTA show a spot sign that disappeared at the 3-hour follow-up dCTA (e) following INR correction with PCC. The hematoma volume at $3 \mathrm{~h}$ was $67.2 \mathrm{ml}$. f Follow-up non-contrast CT at 3 days shows that the hematoma volume decreased to $52 \mathrm{ml}$. 


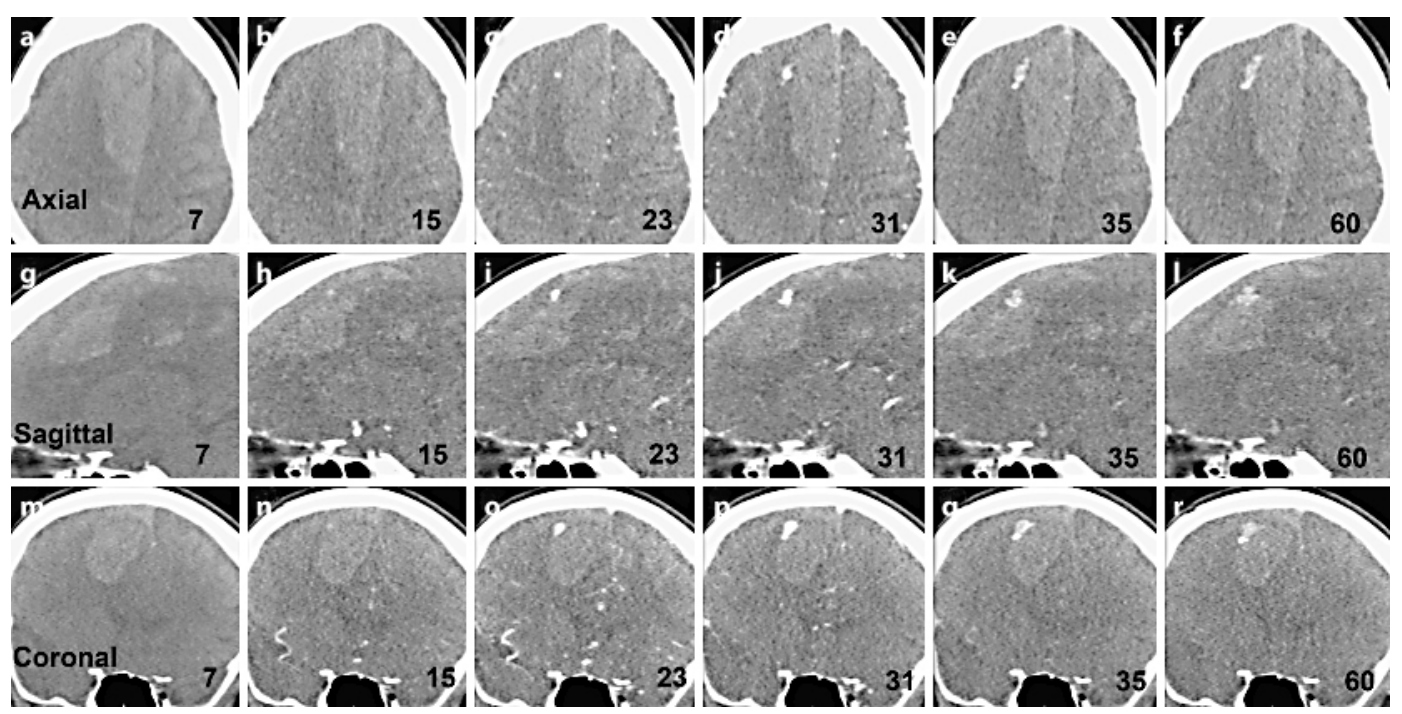

Fig. 2. This figure illustrates time series of spot sign evolution (time from the start of contrast injection is depicted on the lower right hand corner of each image in seconds). Each row shows the spot sign in axial $(\mathbf{a}-\mathbf{f})$, sagittal $(\mathbf{g}-\mathbf{I})$ and coronal $(\mathbf{m}-\mathbf{r})$ plane. The spot appeared at $15 \mathrm{~s}$ following start of contrast injection and steadily increased in size. The maximum density was seen around $31 \mathrm{~s}$; after that, the average density reduced and the margins became ill-defined, consistent with dispersion of contrast within the hematoma. 


\section{References}

1 Kim J, et al: Contrast extravasation on CT predicts mortality in primary intracerebral hemorrhage. AJNR Am J Neuroradiol 2008;29:520-525.

$\checkmark 2$ Becker KJ, et al: Extravasation of radiographic contrast is an independent predictor of death in primary intracerebral hemorrhage. Stroke 1999;30:2025-2032.

-3 Brott T, et al: Early hemorrhage growth in patients with intracerebral hemorrhage. Stroke 1997;28:1-5.

-4 Wada R, et al: CT angiography 'spot sign' predicts hematoma expansion in acute intracerebral hemorrhage. Stroke 2007;38:1257-1262.

5 Chakraborty S, et al: Dynamic nature of the CT angiographic 'spot sign'. Br J Radiol 2010;83:e216-e219.

6 Mayer SA, et al: Efficacy and safety of recombinant activated factor VII for acute intracerebral hemorrhage. N Engl J Med 2008;358:2127-2137.

7 Aviv RI, et al: Contrast extravasation predicts hematoma growth: where to now? AJNR Am J Neuroradiol 2008;29:E80

8 Goldstein JN, et al: Contrast extravasation on CT angiography predicts hematoma expansion in intracerebral hemorrhage. Neurology 2007;68:889-894.

19 Delgado Almandoz JE, et al: The spot sign score in primary intracerebral hemorrhage identifies patients at highest risk of in-hospital mortality and poor outcome among survivors. Stroke 2010;41:54-60.

10 Ederies A, et al: Postcontrast CT extravasation is associated with hematoma expansion in CTA spot negative patients. Stroke 2009;40:1672-1676.

11 Hallevi $\mathrm{H}$, et al: The spot sign in intracerebral hemorrhage: the importance of looking for contrast extravasation. Cerebrovasc Dis 2010;29:217-220.

12 Leira R, et al: Early neurologic deterioration in intracerebral hemorrhage: predictors and associated factors. Neurology 2004;63:461-467.

13 Davis SM, et al: Hematoma growth is a determinant of mortality and poor outcome after intracerebral hemorrhage. Neurology 2006;66:1175-1181.

14 Dowlatshahi D, et al: Defining hematoma expansion in intracerebral hemorrhage: relationship with patient outcomes. Neurology 2011;76:1238-1244. 